\title{
Apolipoprotein E Genotype and Changes in Serum Lipids and Maximal Oxygen Uptake With Exercise Training
}

\author{
Paul D. Thompson, Gregory J. Tsongalis, Richard L. Seip, Cherie Bilbie, Mary Miles, Robert Zoeller, \\ Paul Visich, Paul Gordon, Theodore J. Angelopoulos, Linda Pescatello, Linda Bausserman, and Niall Moyna
}

\begin{abstract}
Physical activity improves lipid levels by altering triglyceride (TG) metabolism. Apolipoprotein E (Apo E) facilitates TG clearance by mediating lipoprotein binding to hepatic receptors, but Apo $E$ also has less defined roles in skeletal muscle and nervous tissue. This study examined if variants in Apo E genotype affect the lipid and physiologic response to exercise training. Seven centers genetically screened 566 individuals to recruit 120 subjects into 6 gender-specific cohorts equal for the most common Apo E genotypes: E2/3, E3/3, and E3/4. Anthropometics, exercise capacity ( $\left.\mathrm{Vo}_{2} \mathrm{max}\right)$, serum lipids, and post heparin (PH) plasma lipase activities were measured before and after 6 months of supervised exercise training. Difference in the response $(\Delta)$ to training among the Apo $E$ genotypes was the primary outcome variable. Differences in pretraining serum lipids among the Apo E genotypes mimicked those observed in population studies: TGs were slightly higher in E2/3 subjects, whereas low-density lipoprotein (LDL)-cholesterol (C) was lower ( $P=$ not significant [NS]). TGs decreased $11 \%$ with training for the entire cohort $(P<.0001)$ and $7 \%, 12 \%$, and $14 \%$ for the Apo E 2/3,3/3 and 3/4 groups, respectively $(P=N S$ for $\Delta)$. LDL-C did not change in the entire cohort, but decreased slightly in the $2 / 3$ and $3 / 3$ subjects and increased $4 \%$ in the $3 / 4 \mathrm{group}(P=$ NS for $\Delta)$. High-density lipoprotein (HDL)-C increased $2 \%$ for the entire cohort $(P=.06)$ due to a $6 \%$ increase in the $3 / 3$ group $(P=.07$ for $\Delta)$. Total cholesterol (TC)/HDL and LDL/HDL decreased with training in the $2 / 3$ and $3 / 3$ groups, but increased in the $3 / 4$ subjects and these responses differed among the genotypes $(P<.05$ for $\Delta)$. $\mathrm{Vo}_{2}$ max increased $9 \%$ to $10 \%$ for the entire cohort, but only $5 \%$ in the $3 / 3$ subjects versus $13 \%$ in the $2 / 3$ and $3 / 4$ groups and these differences were significantly different among the genotypes $(P<.01$ for $\Delta)$. This is the first prospective study to demonstrate that the serum lipid response to exercise training differs by Apo $E$ genotype in a pattern consistent with known metabolic differences among the variants. Surprisingly, Apo E genotype also affected the increase in aerobic capacity produced by exercise training possibly via undefined effects on nerve and skeletal muscle function. (C) 2004 Elsevier Inc. All rights reserved.
\end{abstract}

$\mathbf{E}$ NDURANCE ATHLETES have high-density lipoprotein (HDL)-cholesterol (C) concentrations approximately 20 $\mathrm{mg} / \mathrm{dL}$ or $40 \%$ higher than sedentary subjects and triglyceride (TG) levels $40 \mathrm{mg} / \mathrm{dL}$ or $45 \%$ lower. $^{1}$ Physical activity and HDL appear to be linked via HDL's role in TG metabolism. ${ }^{2}$ Free fatty acids are a major energy source during prolonged exertion and are derived from intramuscular and circulating TG by the actions of hormone-sensitive and lipoprotein lipase (LPL), respectively. This delipidation of chylomicrons and very-low-density lipoproteins (VLDL) produces excess surface cholesterol that is transferred to the HDL particle. ${ }^{3,4}$ Consequently, the intravascular hydrolysis of TG-rich lipoproteins could simultaneously replace intramuscular fat used during exercise, reduce TGs and increase HDL-C.

Changes in serum lipids with exercise training are often small and individually variable, limiting the role of exercise in treating lipid abnormalities. A meta-analysis of 59 exercise training studies reported an average increase in HDL-C of only $2 \mathrm{mg} / \mathrm{dL} .{ }^{5}$ Furthermore, several studies recently reviewed ${ }^{6}$ suggest that exercise is less effective in increasing HDL and altering TG metabolism in individuals with initially elevated TGs and low HDL. Such observations suggest that individual differences contribute to the variability in the exercise response.

Apolipoprotein E (Apo E) mediates the binding of VLDL and intermediate-density lipoproteins (IDL) to the hepatic lowdensity lipoprotein (LDL) and VLDL receptors ${ }^{7}$ and the binding of chylomicron remnants to the chylomicron remnant receptor, thereby facilitating TG clearance. ${ }^{8}$ Apo E has 299 amino acids with a binding region between amino acids 140 and 160. There are 3 common allelic variants $\left(\epsilon_{2}, \epsilon_{3}, \epsilon_{4}\right)$, producing 3 protein polymorphisms: E2, E3 and E4. Apo E3 is the most common or "wild-type" and contains a cysteine at amino acid 112 and an arginine within the binding region at amino acid $158 .{ }^{9}$ The E4 variant contains an arginine at the 112 site and has normal or enhanced binding capacity, whereas the E2 variant contains a cysteine substitution within the binding region at amino acid 158 and has only $1 \%$ to $2 \%$ of normal binding capacity. ${ }^{9}$ A meta-analysis of 45 studies from 17 countries and including 14,799 subjects found population frequencies of $0.6 \%$ for $\mathrm{E} 2 / \mathrm{E} 2,10.7 \%$ for E2/ E3, $62.4 \%$ for E3/E3, $1.6 \%$ for E2/E4, $22.3 \%$ for E3/E4, and $2.3 \%$ for E4/E4. ${ }^{10}$ Given the relationship between fat metabolism and the lipid response to exercise training as well as the importance of Apo E in TG metabolism, we speculated that variation in Apo E would contribute to the variability in individual response to exercise training. The present study tested this hypothesis.

From the Hartford Hospital, Hartford, CT; Montana State University, Bozeman, MT; Florida Atlantic University, Davie, FL; Central Michigan University, Mt Pleasant, MI; West Virginia University, Morgantown, WV; University of Central Florida, Orlando, FL; University of Connecticut, Storrs, CT; Lifespan Health System, Brown University, Providence RI; and Dublin City University, Dublin, Ireland.

Submitted April 19, 2003; accepted September 3, 2003.

Supported by grants from the Donaghue Foundation, Hartford, CT and Research Administration, Hartford Hospital.

Address reprint requests to Paul D. Thompson, MD, Cardiology

Division, Hartford Hospital, 80 Seymour St, Hartford, CT 06102.

(C) 2004 Elsevier Inc. All rights reserved.

0026-0495/04/5302-0013\$30.00/0

doi:10.1016/j.metabol.2003.09.010 


\section{MATERIALS AND METHODS}

\section{Study Overview}

This study was conducted by the Exercise and Genetics Collaborative Research Group, a consortium of investigators at seven institutions. Informed consent was obtained from 566 individuals who were screened by genotype to create 6 cohorts of men and women with equal number of the 3 most common Apo E genotypes: Apo E 2/3, 3/3, and 3/4. A total of 174 subjects initiated exercise training and 120 completed the 6-month program. Investigators were unaware of subjects' genotype and only informed whether or not a subject qualified by genotype for inclusion. Apo E genotypes were intentionally distributed among the sites by the coordinating center (Hartford Hospital) to prevent subtle differences in the training regimens among sites from affecting the results. Four men and 3 women who completed the study were excluded from analysis because their TG levels were greater than $400 \mathrm{mg} / \mathrm{dL}$, invalidating the Friedewald calcuation of LDL-C $(\mathrm{n}=4)$ or because their pretraining HDL-C was greater than $3 \mathrm{SD}$ from the overall mean of $48.5 \pm 13.3 \mathrm{mg} / \mathrm{dL}$.

\section{Subjects}

Subjects were recruited if they were healthy and without orthopedic problems, nonsmokers, physically inactive, $\geq 18$ and less than 70 years old, and consumed fewer than 2 alcoholic beverages daily. Subjects were considered physically inactive if they participated in vigorous activity fewer than 4 times per month for the prior 6 months. We did not recruit individuals with a body mass index (BMI) greater than 31 since caloric restriction reduces HDL-C, ${ }^{11}$ and we sought to avoid subjects who might restrict their caloric intake during lipid measurement. Subjects underwent a medical history, physical examination, and a maximal exercise test to detect unreported abnormalities and occult coronary artery disease. Subjects were reimbursed $\$ 250$ at the end of the study.

\section{Apo E Genotype Determination}

DNA was extracted from leukocytes and Apo E variants determined using standard techniques. ${ }^{12}$

\section{Serum Lipid, Lipoprotein, and Post Heparin Lipoprotein Lipase, and Hepatic TG Lipase Activity Measurements}

Two serum samples were obtained after a 12-hour fast before the start and after 6 months of exercise training. Post-training samples were obtained within 24 hours of the penultimate and final exercise training session. Lipid levels in women before and after training were obtained within 10 days of the onset of menses to avoid variations in lipoprotein values. ${ }^{13}$ Serum was separated from plasma and frozen at $-70^{\circ} \mathrm{C}$ until analyzed by the Lipid Research Laboratory, Lifespan Health System, Brown University, Providence RI. All samples from an individual subject were analyzed in the same analysis run at the end of the study to minimize the effect of laboratory variation. Total cholesterol, TGs, HDL-C, $\mathrm{HDL}_{2}$ and $\mathrm{HDL}_{3}$ subfractions, Apo $\mathrm{AI}$ and $\mathrm{B}$, and post heparin (PH) lipoprotein lipase (LPLA) and hepatic TG lipase (HTGLA) were determined using standard techniques. ${ }^{14}$ Coefficients of variation for lipid measurements in this laboratory are as follows: total cholesterol (TC) $(0.8 \%)$, TGs $(1.5 \%)$, HDL-C $(2.0 \%), \mathrm{HDL}_{3}$ subfraction $(6.3 \%)$, Apo AI $(2.6 \%)$ and B (1.8\%), and PH LPLA (14\%) and HTGLA $(12 \%)$.

\section{Anthropometric Measurements}

Body weight and height were measured using balance beam scales and wall-mounted tape measures. Skinfold thickness was measured on the right side of the body using calipers to estimate percent body fat in men ${ }^{15}$ and women. ${ }^{16}$

\section{Maximal Exercise Capacity}

Subjects underwent 2 pre- and 1 post-training maximal treadmill exercise tests using the modified Astrand protocol. ${ }^{17}$ The first pretraining test was designed to detect occult ischemia and to familiarize subjects with the measurement protocol, but was not used in data analysis. Blood pressure and 12-lead electocardiogram, as well as expired oxygen, carbon dioxide, and ventilatory volume were measured. Maximal oxygen uptake $\left(\mathrm{VO}_{2} \mathrm{max}\right)$ was defined as the average of the 2 highest consecutive 30 -second values at peak exercise.

\section{Dietary Control Procedures}

Subjects were requested to maintain their usual dietary composition throughout the study. Dietary calories and composition were assessed by random, 24-hour dietary recalls. ${ }^{18,19}$ Trained dieticians called the subjects by telephone on 1 weekday and 1 weekend day before the start and during the last month of exercise training. Results from the 2 calls were averaged to estimate dietary intake.

\section{Exercise Program}

Subjects underwent a progressive, supervised exercise training program. The duration of each exercise session was increased from 15 to 40 minutes during the first 4 weeks. Subjects exercised between $60 \%$ and $85 \%$ of their maximal exercise capacity $\left(\mathrm{VO}_{2} \max \right)$ based on their predetermined maximal heart rate. Once subjects could perform 40 minutes of exercise, they continued this duration of exercise 4 days a week for an additional 5 months for a total of 6 months of participation. Subjects also participated in 5 minutes of warm-up and cool-down so that each workout required 50 minutes. Treadmill exercise was the primary mode of training, but subjects were able to use a variety of training modalities including treadmills, stationary cycles, cross-country ski machines, stair steppers, and rowing machines for variety and to minimize orthopedic injury.

\section{Exercise Energy Expenditure}

Weekly exercise energy expenditure expressed as kilocalories per week was estimated from the average heart rates recorded for exercise sessions of that week. From individual plots of $\mathrm{VO}_{2}$ versus heart rate created from pretraining maximal exercise test data, we estimated the $\mathrm{VO}_{2}$ corresponding to the training exercise heart rate intensity and multiplied that $\mathrm{VO}_{2}$ by training session duration to obtain total oxygen consumption for each bout. Each liter of oxygen was assumed to represent $5 \mathrm{kcal}$ of energy expenditure.

\section{Data Analysis and Sample Size Estimation}

We estimated the required sample size by 3 methods. First, we used the TG change by Apo E genotype observed in a preliminary study 20 and estimated that 18 subjects of each gender would be required in each genotype group. Second, we used the percent decrease in HDL-C by Apo E genotype observed by others during fasting ${ }^{21}$ to estimate that 19 subjects would be required in each group. Third, we postulated that the increase in HDL-C in the Apo E2/E3 subjects would be $2 \mathrm{mg} / \mathrm{dL}$ or $50 \%$ greater than the $10 \%$ average increase in HDL-C we previously observed for unselected subjects after exercise training ${ }^{14}$ and that the changes in the other Apo E groups would be 50\% less. Using this approach, 16 subjects would be required in each group. Consequently, we sought to recruit 20 subjects per group.

\section{Data Analysis}

TGs were log-transformed to improve normality. Paired $t$ tests were used to test training changes in the entire cohort for significance. Univariate analyses of variance (ANOVAs) were used to compare Apo E groups for differences at baseline and differences in their responses 
Table 1. Baseline Physiological Characteristics, and Change With Training $(\Delta)$, in All Subjects and by Apo E Genotype (means \pm SD)

\begin{tabular}{|c|c|c|c|c|c|c|c|c|c|c|}
\hline & \multicolumn{2}{|c|}{ All Subjects } & \multicolumn{2}{|c|}{$2 / 3(n=40)$} & \multicolumn{2}{|c|}{$3 / 3(n=43)$} & \multicolumn{2}{|c|}{$4 / 3(n=37)$} & \multirow{2}{*}{$\begin{array}{c}\text { ANOVA on } \\
\text { Baseline, } \\
P \text { Value }\end{array}$} & \multirow{2}{*}{$\begin{array}{c}\text { ANOVA on } \\
\text { Changes, } \\
P \text { Value }\end{array}$} \\
\hline & Pre & $\Delta$ & Pre & $\Delta$ & Pre & $\Delta$ & Pre & $\Delta$ & & \\
\hline Age $(y r)$ & $39 \pm 11$ & - & $39 \pm 10$ & - & $40 \pm 12$ & - & $36 \pm 10$ & - & .389 & - \\
\hline Body weight (kg) & $81.2 \pm 18.2$ & $-1.2 \pm 3.3 \dagger$ & $81.7 \pm 17.2$ & $-1.0 \pm 3.2$ & $80.6 \pm 16.9$ & $-1.2 \pm 3.5$ & $81.3 \pm 21.0$ & $-1.4 \pm 3.3$ & .957 & .859 \\
\hline$\%$ Body fat & $22.7 \pm 6.1$ & $-0.9 \pm 2.8^{*}$ & $23.3 \pm 4.9$ & $-0.7 \pm 2.4$ & $22.2 \pm 6.5$ & $-1.0 \pm 2.9$ & $22.8 \pm 6.8$ & $-1.1 \pm 3.1$ & .714 & .825 \\
\hline WHR & $0.84 \pm 0.09$ & $-0.01 \pm 0.04$ & $0.83 \pm 0.10$ & $-0.01 \pm 0.05$ & $0.84 \pm 0.09$ & $-0.00 \pm 0.03$ & $0.84 \pm 0.09$ & $-0.01 \pm .03$ & .885 & .421 \\
\hline \multicolumn{11}{|l|}{$\mathrm{Vo}_{2} \max$} \\
\hline$\left(\mathrm{L} \mathrm{O}_{2} / \mathrm{min}\right)$ & $2.55 \pm 0.79$ & $0.24 \pm 0.33 \dagger$ & $2.49 \pm 0.86$ & $0.30 \pm 0.39 \neq$ & $2.62 \pm 0.75$ & $0.09 \pm 0.25 \ddagger \S$ & $2.57 \pm 0.76$ & $0.34 \S \pm 0.32$ & .753 & .001 \\
\hline \multicolumn{11}{|l|}{$\mathrm{Vo}_{2} \max$} \\
\hline$(\mathrm{mL} \mathrm{O} / 2 / \mathrm{kg} / \mathrm{min})$ & $32.0 \pm 7.8$ & $3.3 \pm 4.2 \dagger$ & $30.4 \pm 7.8$ & $4.1 \pm 5.1 \neq$ & $32.9 \pm 7.7$ & $1.6 \pm 2.7 \ddagger \S$ & $32.6 \pm 7.7$ & $4.3 \S \pm 3.8$ & .282 & .004 \\
\hline
\end{tabular}

Abbreviations: WHR, waist-to-hip ratio. $\mathrm{VO}_{2} \max$, maximal oxygen uptake.

${ }^{*} P<.001,+P<.0001$, significant $\Delta$ within group, based on paired $t$ test.

¥Significant difference in $\Delta$ between E2/E3 and E3/E3, $P<.05$.

§Significant difference in $\Delta$ between E4/E3 and E3/E3, $P<.01$, based on Bonferroni-corrected pairwise differences and Tukey's HSD or Scheffé post hoc tests.

to exercise training. Tukey honest significant difference (HSD) or Scheffé post hoc tests were used to test for differences between Apo E groups when $F$ ratios were significant. Covariates, selected on the basis of correlations and partial correlations, were used in ANCOVA. Bonferroni corrected $t$ tests were used as post hoc tests only when analysis of covariance (ANCOVA) $F$ ratios were significant. Significance levels were 2-sided with alpha $=0.05$.

\section{RESULTS}

\section{Effect of Exercise Training on Anthropometric Parameters}

The age of the subjects was $39 \pm 11$ years (mean \pm SD) for the entire cohort and did not differ by Apo E genotype (Table 1). Body weight and estimated percent body fat was not different at baseline among the 3 Apo E genotypes. Both body weight and body fat decreased slightly, but significantly, with exercise training in the entire cohort. These changes were not different among the Apo E genotypes.

\section{Adherence to Exercise Training}

Exercise adherence was high in all 3 genotype groups with subjects in the Apo E 2/3, 3/3, and 3/4 groups attending 91.7\%, $93.8 \%$, and $94.8 \%$ of the total exercise sessions, respectively. Exercise training intensity measured as percent of maximal heart rate was also similar among the genotypes at $74 \%, 76 \%$, and $76 \%$, respectively. There were 56 dropouts and 121 finishers and no difference in Apo E genotype distribution between the drop outs and finishers. Weekly exercise energy expenditure during most of the study averaged 1,200 to 1,400 kcal (Table 2). Each institution trained an average of 16 subjects with a range of 10 to 23 . The training regimen was identical across sites and every bout was supervised. Heart rates were recorded at all sessions to monitor intensity and did not vary between sites.

\section{Effect of Exercise Training on Exercise Capacity}

$\mathrm{VO}_{2}$ max did not differ among the Apo $\mathrm{E}$ groups before training and increased $9 \%$ to $10 \%$ for the entire cohort when expressed in absolute values or relative to body weight $(P<$ .0001). The increase in $\mathrm{VO}_{2} \max$ was significantly less in the Apo E 3/3 subjects than in either of the other 2 Apo E genotypes $(P<.01)$ (Fig 1$)$. This smaller increase in $\mathrm{VO}_{2} \mathrm{max}$ in the Apo E 3/3 group persisted after adjustment for baseline $\mathrm{Vo}_{2}$, and other parameters, including age, baseline BMI, and baseline waist-to-hip ratio.

\section{Effect of Exercise Training on Lipid Values}

Baseline TC did not differ among the Apo E genotypes (Table 3). TC was unchanged by exercise training in the entire cohort and in the Apo E groups. These results were not altered by adjustment for a number of covariates, including baseline cholesterol, age, BMI, and change in $\mathrm{VO}_{2}$ max.

Average TGs before training were $5 \%$ and $15 \%$ higher in the Apo E 2/3 than in the Apo E 3/3 and 3/4 groups, respectively $(P=$ not significant $[\mathrm{NS}])$. TGs decreased $11 \%$ for the entire cohort $(P<.0001)$. This decrease was $7 \%, 12 \%$, and $14 \%$ in the Apo E 2/3, 3/3, and 3/4 subgroups, respectively, but these changes were not statistically different among the Apo E genotypes (Fig 2). Results were not altered by adjusting for covariates, including baseline TGs, age, BMI, and change in $\mathrm{VO}_{2} \max$.

Pretraining LDL-C was 9\% lower in the Apo E 2/3 group compared to the other 2 genotypes $(P=\mathrm{NS})$. LDL-C for the entire cohort was unchanged by exercise training. This result was due to small decreases in LDL in the Apo E 2/3 and 3/3 groups and a $4 \%$ increase in the Apo E 3/4 subjects. These differences among the Apo E genotypes were not statistically

Table 2. Intensity, Duration, and Weekly Energy Expenditure Associated With Exercise Training Program

\begin{tabular}{|c|c|c|c|c|c|c|c|}
\hline \multirow[b]{2}{*}{ Variable } & \multicolumn{7}{|c|}{ Training Week } \\
\hline & 1 & 4 & 8 & 12 & 16 & 20 & 24 \\
\hline Intensity (bpm) & $128.1 \pm 19.3$ & $128.1 \pm 19.3$ & $137.5 \pm 18.8$ & $140.1 \pm 14.7$ & $140.3 \pm 15.4$ & $140.4 \pm 14.9$ & $140.6 \pm 15.2$ \\
\hline Duration/bout (min) & $31.6 \pm 13.1$ & $40.8 \pm 12.0$ & $41.6 \pm 14.3$ & $42.0 \pm 13.2$ & $43.6 \pm 13.4$ & $41.2 \pm 12.8$ & $35.7 \pm 13.6$ \\
\hline $\mathrm{kcal} / \mathrm{wk}$ & $710 \pm 497$ & $1,346 \pm 643$ & $1,332 \pm 704$ & $1,396 \pm 661$ & $1,465 \pm 688$ & $1,413 \pm 665$ & $1,232 \pm 671$ \\
\hline
\end{tabular}

Abbreviation: bpm, beats per minute. 


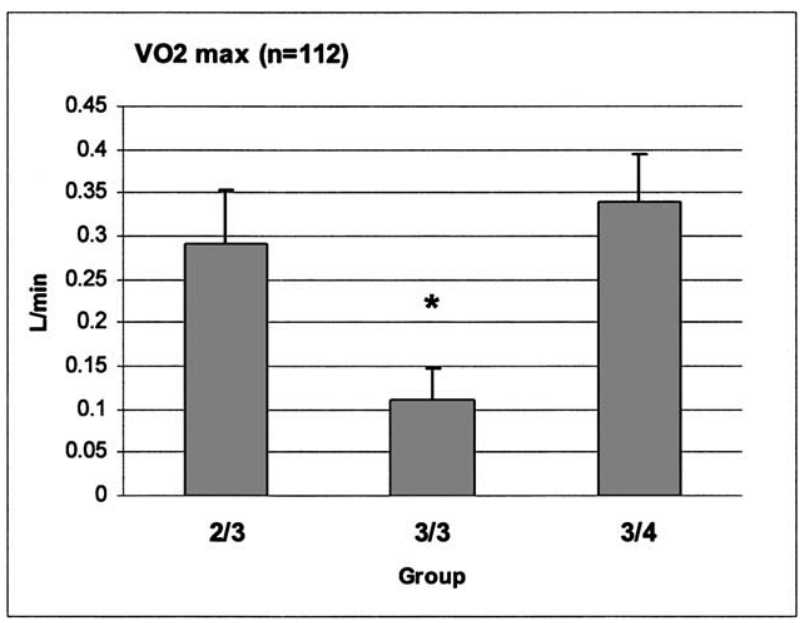

Fig 1. Change in $\mathrm{Vo}_{2} \mathrm{max}$ with training. ${ }^{*}$ Change differed significantly in E3/E3 $v$ E2/E3 $(P<.05)$ and E3/E4 $(P<.01)$.

significant even after adjustment for baseline LDL, age, and BMI $(P=.10$, by ANCOVA).

Pretraining Apo B was also 9\% lower in the Apo E 2/3 subjects compared to the other Apo E groups $(P=\mathrm{NS})$. Apo B decreased $4 \%$ with exercise training in the Apo 2/3 subjects and increased slightly in the other Apo E groups. After adjustment for baseline Apo B levels, age, and BMI, differences in the Apo $\mathrm{B}$ response to training among the Apo E genotypes approached, but did not achieve, statistical significance $(P=.07$, by ANCOVA).

Baseline HDL-C did not differ among the three Apo E genotypes. HDL-C increased $1 \pm 6 \mathrm{mg} / \mathrm{dL}$ with training in the entire cohort $(P=.06)$. This effect was primarily due to a $3-\mathrm{mg} / \mathrm{dL}$ increase in the Apo E 3/3 subjects, the group that achieved the smallest increase in $\mathrm{VO}_{2}$ max, whereas HDL was unchanged in the Apo E 2/3 and 4/4 subjects. The difference in
HDL response among the 3 genotypes approached, but did not achieve, statistical significance $(P=.07)$. This result was not affected by adjustment for covariates including baseline HDL, age, and BMI $(P<.06)$. The HDL-C subfractions, $\mathrm{HDL}_{2}$ and $\mathrm{HDL}_{3}$, did not differ among the Apo E genotypes at baseline or in their response to exercise training. Apo A1 concentrations were also not different among the Apo E groups at baseline or in their response to exercise training, although the increase in the Apo E 3/3 group was larger than the change in the other Apo E groups $(P=\mathrm{NS})$.

The ratios of TC/HDL-C, LDL-C/HDL-C, and Apo B/AI were not different among the Apo E genotypes before training. These ratios decreased slightly with exercise training in the entire cohort, but only the change in Apo B/A1 was statistically significant. The response of these ratios to exercise training was significantly different among the Apo E genotypes due to small decreases in the Apo E2/3 and 3/3 groups and increases in the Apo $3 / 4$ subjects $(P<.05$ for all $)$. This difference remained significant after adjustment for covariates including baseline lipid ratios, BMI, and age.

HTGLA and LPLA values did not differ among the genotypes at baseline or in their response to exercise training. For the entire cohort HTGLA decreased slightly but significantly $(-5 \%, P<.01)$, whereas a similar magnitude increase in LPLA was not statistically significant $(+5 \%, P=\mathrm{NS})$.

\section{Effect of Gender on the Response to Exercise Training}

Men and women at baseline showed the expected differences in anthropometric, exercise capacity, and lipid parameters (Table 4). Despite these baseline differences, the response to exercise training was generally similar for the men (Tables 5 and 6) and women (Tables 7 and 8) and mirrored the changes for the entire cohort. Differential changes among the Apo E subgroups were also similar to those in entire study population.

Body weight decreased slightly but significantly for both genders, whereas $\mathrm{VO}_{2}$ max increased. The increase in $\mathrm{Vo}_{2} \max$

Table 3. Baseline (Pre) Lipid $\left(\mathrm{mg} \cdot \mathrm{dL}^{-1}\right)$, PH LPLA $\left(\mu \mathrm{mol}\right.$ FFA $\left.\cdot \mathrm{mL}^{-1} \cdot \mathrm{h}^{-1}\right)$, and Change With Training $(\Delta)$ Before and After Exercise Training for All Subjects and by Apo E Genotype (mean \pm SD)

\begin{tabular}{|c|c|c|c|c|c|c|c|c|c|c|}
\hline & \multicolumn{2}{|c|}{$\begin{array}{c}\text { Subjects TG }<400 \mathrm{mg} / \mathrm{dL} \\
\mathrm{HDL}<90 \mathrm{mg} / \mathrm{dL}\end{array}$} & \multicolumn{2}{|c|}{$2 / 3(n=38)$} & \multicolumn{2}{|c|}{$3 / 3(n=39)$} & \multicolumn{2}{|c|}{$4 / 3(n=36)$} & \multirow{2}{*}{$\begin{array}{c}\text { ANOVA } \\
\text { on } \\
\text { Baseline, } \\
P \text { Value }\end{array}$} & \multirow{2}{*}{$\begin{array}{c}\text { ANOVA or } \\
\text { ANCOVA on } \\
\text { Changes, } P \\
\text { Value }\end{array}$} \\
\hline & Pre & $\Delta$ & Pre & $\Delta$ & Pre & $\Delta$ & Pre & $\Delta$ & & \\
\hline TG & $127.0 \pm 63.3$ & $-13.7 \pm 40.2$ & $134.3 \pm 71.0$ & $-10.3 \pm 50.8$ & $127.2 \pm 64.6$ & $-14.9 \pm 37.9$ & $119.1 \pm 53.4$ & $-15.9 \pm 29.8$ & .492 & .794 \\
\hline LDL-C & $129.9 \pm 33.2$ & $0.6 \pm 20.2$ & $121.4 \pm 31.7$ & $-0.32 \pm 18.4$ & $135.3 \pm 30.6$ & $-2.3 \pm 20.0$ & $132.8 \pm 36.6$ & $4.5 \pm 22.1$ & .183 & .335 \\
\hline Apo B & $119.9 \pm 28.9$ & $-1.3 \pm 13.9$ & $112.0 \pm 28.0$ & $-5.40 \pm 13.1$ & $123.6 \pm 28.4$ & $0.50 \pm 13.0$ & $124.4 \pm 29.4$ & $1.2 \pm 13.1$ & .149 & .070 \\
\hline HDL-C & $47.4 \pm 11.1$ & $1.1 \pm 6.0$ & $48.7 \pm 10.6$ & $0.2 \pm 5.7$ & $47.1 \pm 11.3$ & $2.9 \pm 5.9$ & $46.4 \pm 11.4$ & $0.2 \pm 5.9$ & .726 & .062 \\
\hline $\mathrm{HDL}_{3}-\mathrm{C}$ & $33.3 \pm 5.9$ & $0.7 \pm 4.9$ & $33.3 \pm 5.0$ & $0.7 \pm 4.4$ & $33.7 \pm 6.1$ & $1.0 \pm 5.8$ & $32.9 \pm 6.7$ & $0.2 \pm 4.4$ & .752 & .811 \\
\hline Apo Al & $155.2 \pm 29.9$ & $2.0 \pm 19.8 \dagger$ & $162.1 \pm 31.0$ & $1.4 \pm 17.0$ & $156.2 \pm 32.4$ & $3.5 \pm 14.0$ & $146.9 \pm 24.0$ & $0.9 \pm 26.9$ & .106 & .784 \\
\hline $\mathrm{TC} / \mathrm{HDL}$ & $4.51 \pm 1.35$ & $-0.16 \pm 0.63$ & $4.25 \pm 1.32$ & $-0.16 \pm 0.57$ & $4.6 \pm 1.30$ & $-0.32 \pm 0.57 \|$ & $4.6 \pm 1.44$ & $0.02 \pm 0.57 \|$ & .433 & $.033^{*}$ \\
\hline LDL/HDL & $2.91 \pm 1.09$ & $-0.08 \pm 0.55$ & $2.65 \pm 1.02$ & $-0.13 \pm 0.50$ & $3.04 \pm 1.04$ & $-0.21 \pm 0.49 \#$ & $3.06 \pm 1.20$ & $0.10 \pm 0.49 \#$ & .228 & $.015^{*}$ \\
\hline Apo B/Al & $0.81 \pm 0.28$ & $-0.02 \pm 0.154 \dagger$ & $0.73 \pm 0.28$ & $-0.06 \pm 0.14$ & $0.82 \pm 0.26$ & $-0.02 \pm 0.14$ & $0.87 \pm 0.29$ & $0.02 \pm 0.14$ & .097 & $.046 *$ \\
\hline HTGLA & $18.2 \pm 8.3$ & $-1.0 \pm 4.4 \dagger$ & $19.3 \pm 9.0$ & $-0.3 \pm 5.0$ & $16.8 \pm 8.4$ & $-1.3 \pm 3.6$ & $18.5 \pm 7.4$ & $-1.4 \pm 4.6$ & .362 & .527 \\
\hline PH LPLA & $11.8 \pm 3.7$ & $0.6 \pm 4.3$ & $12.3 \pm 3.1$ & $1.0 \pm 3.5$ & $11.9 \pm 4.0$ & $-0.3 \pm 3.7$ & $11.2 \pm 3.8$ & $1.2 \pm 5.4$ & .473 & .290 \\
\hline
\end{tabular}

NOTE. LDL-C, Apo B, LDL/HDL, TC/HDL, Apo B/Al changes adjusted for baseline lipid value, BMI, and age.

Abbreviations: TC, total cholesterol; TG, triglycerides; LDL, low-density lipoprotein; C, cholesterol; HDL, high-density lipoprotein; Apo, apolipoprotein; HTGLA, post heparin hepatic triglyceride lipase; LPLA, lipoprotein lipase.

${ }^{*} P<.05, \dagger P<.01, \mp P<.001, \S P<.0001$, significance of $\Delta$ within group based on paired $t$ test.

I $\Delta$ in E3/E3 is different from $\Delta$ in E2/E3, $P<0.01$. $\|, \#$ in E3/E3 is different from $\Delta$ in E4/E3, $P<.05$ and $P<.01$, respectively, based on Bonferroni corrected post hoc tests. 

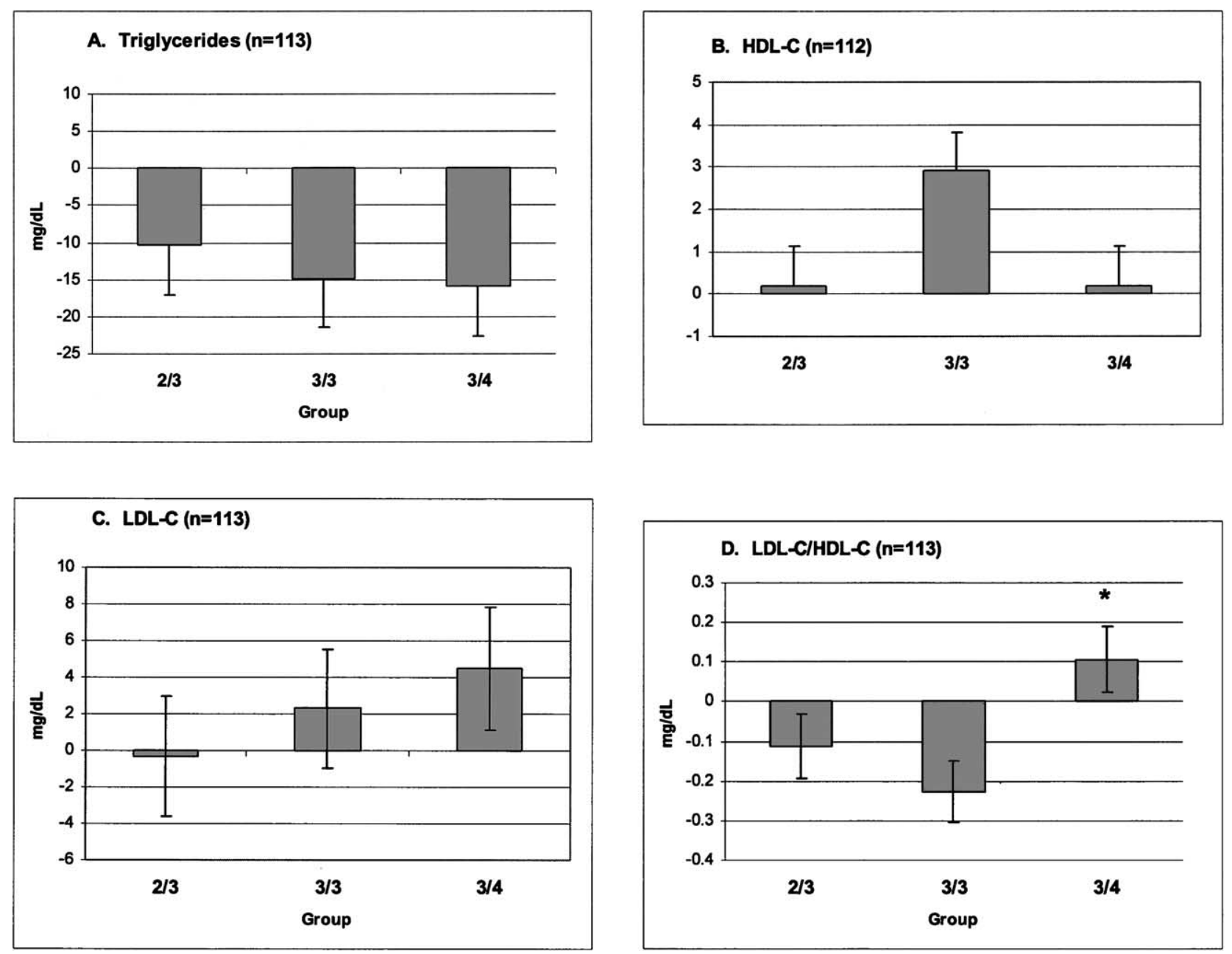

Fig 2. Changes in serum lipids. The bars represent standard errors of the mean. (A) TGs. (B) HDL-C adjusted for baseline HDL-C. Trend toward change in E3/E3 > E2/E3 $(P<.14)$ and E3/E4 $(P<$.10). (C) LDL-C. (D) LDL-C/HDL-C ratio, adjusted for baseline LDL-C and age. *E3/E4 > E3/E3 $(P<.013)$.

was significantly smaller in the Apo E 3/3 men and women than in the other genotypes. TGs decreased significantly in both genders and there were no differences in the TG response among the Apo E genotypes. HDL-C increased significantly after training only in the men. In both genders the increase in HDL-C was larger $(P=\mathrm{NS})$ in the Apo E $3 / 3$ subjects than in the other genotypes despite the smaller increase in $\mathrm{VO}_{2}$ max in the Apo E 3/3 group. LDL-C tended to increase more in the Apo E 3/4 subgroups of both genders $(P=\mathrm{NS})$, but in contrast to the entire study population, this was not reflected in significant changes in Apo B or in the ratios reflecting LDL-C concentration.

HTGLA at baseline was significantly higher in the Apo E 2/3 men than in the other Apo E genotypes. HTGLA decreased significantly with exercise training only in the men. LPLA did not increase significantly with exercise training in either gender, although the women did demonstrate a differential effect among the Apo E genotypes. LPLA decreased in the Apo E 3/3 group but increased in the other 2 genotypes.

\section{Dietary Intake}

Dietary macronutrient composition did not change during training in any subject group or by genotype (data not shown).

\section{DISCUSSION}

The present study is, to our knowledge, the first to examine prospectively the effect of Apo E genotype on the response to exercise training. Given the fact that both Apo E and exercise training have profound effects on lipoprotein metabolism, we postulated that Apo E genotype would affect the lipoprotein response to exercise. The results confirmed this suspicion by demonstrating qualitatively small, but significantly greater, reductions in the ratios of TC/HDL-C, LDL-C/HDL-C, and Apo $\mathrm{B} / \mathrm{A}-\mathrm{I}$ in the Apo E 3/3 subjects, suggesting a greater reduction in cardiovascular risk. In addition, we observed an unexpected smaller increase in exercise capacity in the Apo E 3 homozygotes. 


\begin{tabular}{|c|c|c|}
\hline Variable & $\begin{array}{c}\text { Men } \\
(n=53)\end{array}$ & $\begin{array}{l}\text { Women } \\
(\mathrm{n}=60)\end{array}$ \\
\hline Age & $37 \pm 11$ & $39 \pm 12$ \\
\hline Body weight (kg) & $91.5 \pm 16.6$ & $71.8 \pm 14.3 \ddagger$ \\
\hline Estimated $\%$ body fat & $21 \pm 6$ & $24 \pm 6 \dagger$ \\
\hline Waist/hip & $0.91 \pm 0.06$ & $0.77 \pm 0.05 \S$ \\
\hline $\mathrm{VO}_{2} \max \left(\mathrm{mL} \mathrm{O}{ }_{2} \cdot \mathrm{kg}^{-1} \cdot \mathrm{min}^{-1}\right)$ & $36.4 \pm 7.2$ & $27.9 \pm 6.2 \S$ \\
\hline TC & $202 \pm 40$ & $203 \pm 36$ \\
\hline Triglycerides & $138 \pm 66$ & $117 \pm 60$ \\
\hline LDL-C & $133 \pm 35$ & $127 \pm 32$ \\
\hline HDL-C & $42 \pm 8$ & $52 \pm 11 \S$ \\
\hline $\mathrm{HDL}_{2}-\mathrm{C}$ & $7 \pm 5$ & $15 \pm 9 \S$ \\
\hline $\mathrm{HDL}_{3}-\mathrm{C}$ & $32 \pm 6$ & $34 \pm 6^{*}$ \\
\hline TC/HDL & $5.0 \pm 1.3$ & $4.0 \pm 1.2 \S$ \\
\hline LDL/HDL & $3.3 \pm 1.1$ & $2.6 \pm 1.0 \S$ \\
\hline Apo B & $125 \pm 30$ & $116 \pm 28$ \\
\hline Apo Al & $143 \pm 22$ & $166 \pm 32 \S$ \\
\hline Apo B/Al & $0.90 \pm 0.28$ & $0.73 \pm 0.25 \dagger$ \\
\hline HTGLA & $22.5 \pm 8.4$ & $14.4 \pm 6.1 \S$ \\
\hline HP LPLA & $12.4 \pm 3.7$ & $11.3 \pm 3.6$ \\
\hline
\end{tabular}

${ }^{*} P<.05,+P<.01, \pm P<0.001, \S P<.0001$, significant differences between genders, based on independent $t$ tests.

Large population-based studies have demonstrated differences in serum lipids among the Apo E genotypes. TGs are generally higher in Apo E 2 heterozygotes because of reduced Apo E-mediated binding of TG-rich lipoproteins to the Apo E receptor. ${ }^{10}$ In contrast, TC and LDL-C levels are generally lower in Apo E 2/3 subjects than in the Apo E 3/3 and 3/4 heterozygotes. ${ }^{10}$ Apo E 2's reduced affinity for hepatic binding decreases cholesterol delivery to the hepatocyte. The hepatocyte responds by increasing LDL receptor activity, which reduces LDL-C in the Apo E 2 subjects. ${ }^{22}$ In contrast, LDL-C levels are generally higher in Apo E 3/4 subjects than in Apo 3 homozygotes. ${ }^{10}$ The mechanism is not defined but may ultimately result from reduced binding of Apo E 4 to the HDL particle. ${ }^{9,10}$ Approximately, $30 \%$ of plasma Apo E is associated with HDL. Apo E 4 is less adherent to HDL and is more rapidly transferred to TG-rich particles. More rapid transfer of Apo E
4 from HDL to TG-enriched lipoproteins would promote hepatic delivery of these particles, and increase hepatocyte cholesterol content thereby suppressing hepatic LDL receptor activity and increasing serum LDL-C levels.9,23 The increased LDL-C concentrations may contribute to the observed higher incidence of coronary artery disease in Apo E 4 heterozygotes. ${ }^{24}$

Pretraining lipid levels for the Apo E genotypes in the present study paralleled those noted in population studies. TGs were higher in the Apo E 2 heterozygotes than in the other Apo E groups, whereas LDL-C concentrations were lower. In contrast to larger studies, however, LDL-C was not higher before training in the Apo E 3/4. None of these differences was statistically significant, probably because of the relatively small sample size.

The lipid response to exercise training was significantly different among the Apo E genotypes. LDL-C and Apo B decreased slightly and nonsignificantly in the Apo E 2/3 and 3/3 genotypes, and increased in the Apo E 3/4 group. Fasting TGs decreased with exercise training in all genotypes, suggesting increased conversion of VLDL to LDL. It is possible that this increase in LDL production could not be readily cleared by the Apo E 4 heterozygotes due to the reduced LDL receptor activity discussed above, thereby increasing LDL levels.

There were also differences in the HDL-C response to training. HDL-C increased $1 \mathrm{mg} / \mathrm{dL}$ for the entire cohort, but this increase was primarily due to a $3-\mathrm{mg} / \mathrm{dL}$ increase in the Apo $\mathrm{E}$ 3 homozygotes. The mechanism for this differential response is not clear, but may involve different mechanisms for the different genotypes. The decrease in HTGLA with training in the Apo E $2 / 3$ subjects was only $25 \%$ of that in the other genotypes and the decrease in TGs was $30 \%$ less. Neither difference was statistically significant among the genotypes, but both could have contributed to a smaller increase in HDL-C in the Apo E $2 / 3$ group. On the other hand, smaller increases in HDL-C with training in the Apo E 3/4 individuals may relate to reduced adherence of Apo E 4 to the HDL particle. ${ }^{9,10}$ HDL is cholesterol-enriched in part by the LPL-mediated transfer of cholesterol from VLDL, and this is one of the postulated mechanisms by which exercise training increases HDL-C. ${ }^{2}$ Apo E facilitates the interaction of VLDL with LPL. ${ }^{10}$ Approximately $40 \%$ of

Table 5. Baseline Physiological Characteristics, and Change With Training $(\Delta)$, In All Men and by Apo E Genotype (means \pm SD)

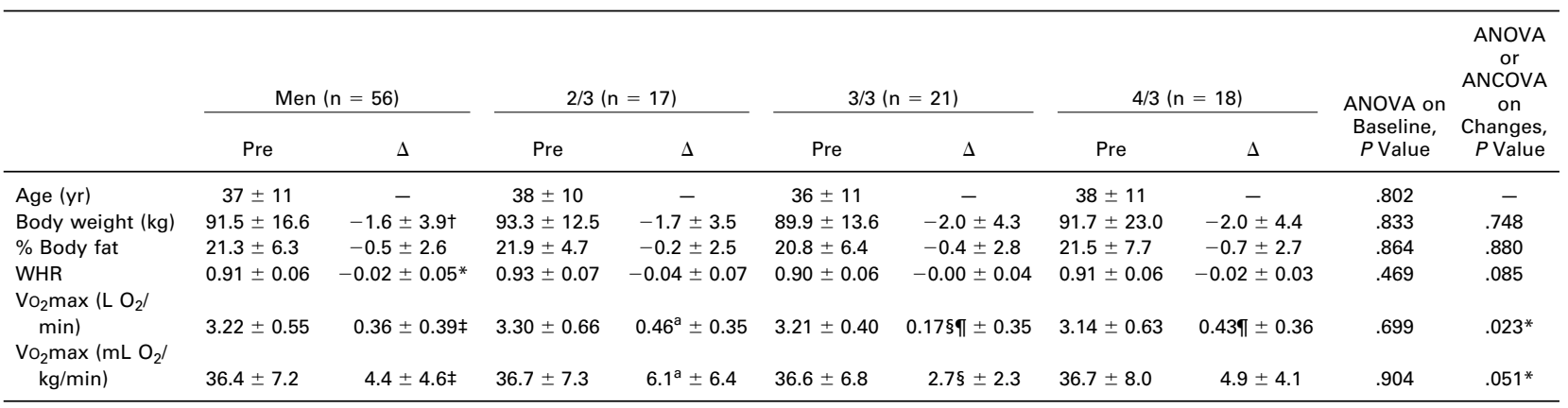

NOTE. $\mathrm{VO}_{2}$ is corrected for baseline value.

${ }^{*} P<.05, \dagger P<.001, \pm P<.0001$, significant $\Delta$ within group, based on paired $t$ test.

§Significant $\Delta$ between E2/E3 and E3/E3, $P<.06$ and $\uparrow \Delta$ between between E3/E3 and E4/E3, $P=.07$, based on Bonferroni corrected pairwise differences, Tukey's HSD, or Scheffé post hoc tests. 
Table 6. Baseline Lipid Concentrations, PH LPLA, and Change With Training $(\Delta)$ in Men (means \pm SD)

\begin{tabular}{|c|c|c|c|c|c|c|c|c|c|c|}
\hline & \multicolumn{2}{|c|}{$\begin{array}{c}\text { Men TG }<400 \text { and } \mathrm{HDL}< \\
90 \mathrm{mg} / \mathrm{dL}\end{array}$} & \multicolumn{2}{|c|}{$2 / 3(n=16)$} & \multicolumn{2}{|c|}{$3 / 3(n=20)$} & \multicolumn{2}{|c|}{$4 / 3(n=17)$} & \multirow{2}{*}{$\begin{array}{l}\text { ANOVA } \\
\text { on Pre, } \\
P \text { Value }\end{array}$} & \multirow{2}{*}{$\begin{array}{c}\text { ANOVA } \\
\text { or } \\
\text { ANCOVA } \\
\text { on } \\
\text { Changes } \\
P \text { Value }\end{array}$} \\
\hline & Pre & $\Delta$ & Pre & $\Delta$ & Pre & $\Delta$ & Pre & $\Delta$ & & \\
\hline TC & $202.4 \pm 39.6$ & $-1.8 \pm 20.7$ & $201.4 \pm 41.4$ & $-3.7 \pm 16.2$ & $204.1 \pm 37.4$ & $-2.9 \pm 26.6$ & $201.3 \pm 42.6$ & $1.3 \pm 17.2$ & .972 & .758 \\
\hline TG & $138.3 \pm 65.6$ & $-17.1 \neq \pm 37.4$ & $149.8 \pm 72.5$ & $-13.7 \pm 42.0$ & $133.9 \pm 70.9$ & $-19.0 \pm 41.1$ & $132.8 \pm 53.9$ & $-17.9 \pm 29.2$ & .711 & .911 \\
\hline LDL-C & $133.0 \pm 34.7$ & $-0.67 \pm 20.5$ & $128.0 \pm 34.2$ & $-2.48 \pm 16.8$ & $136.0 \pm 29.1$ & $-2.9 \pm 16.8$ & $134.1 \pm 42.2$ & $3.59 \pm 21.7$ & .786 & .589 \\
\hline Apo B & $124.9 \pm 29.7$ & $-2.8 \pm 13.8$ & $117.9 \pm 30.4$ & $-6.38 \pm 16.1$ & $125.0 \pm 26.8$ & $-0.48 \pm 13.7$ & $131.2 \pm 32.4$ & $-2.21 \pm 11.3$ & .447 & .439 \\
\hline HDL-C & $41.7 \pm 8.3$ & $2.3 \dagger \pm 5.9$ & $43.5 \pm 7.9$ & $1.50 \pm 4.5$ & $41.4 \pm 8.3$ & $3.7 \pm 6.3$ & $40.6 \pm 9.0$ & $1.29 \pm 6.4$ & .585 & .379 \\
\hline $\mathrm{HDL}_{2}-\mathrm{C}$ & $7.3 \pm 4.8$ & $1.4^{*} \pm 4.8$ & $8.5 \pm 4.1$ & $0.99 \pm 4.4$ & $6.4 \pm 4.6$ & $2.1 \pm 3.9$ & $6.9 \pm 5.6$ & $0.9 \pm 6.2$ & .403 & .733 \\
\hline $\mathrm{HDL}_{3}-\mathrm{C}$ & $32.1 \pm 5.8$ & $0.66 \pm 4.43$ & $32.6 \pm 5.5$ & $0.45 \pm 3.76$ & $32.1 \pm 5.6$ & $1.6 \pm 4.0$ & $31.5 \pm 6.5$ & $-0.23 \pm 4.9$ & .851 & .426 \\
\hline Apo Al & $143.2 \pm 21.9$ & $3.9^{*} \pm 12.6$ & $154.3 \pm 24.1 \|$ & $-0.34 \pm 11.9$ & $143.4 \pm 20.5$ & $4.2 \pm 12.6$ & $132.4 \pm 16.6 \|$ & $7.5 \pm 12.8$ & $.014^{*}$ & .207 \\
\hline $\mathrm{TC} / \mathrm{HDL}$ & $5.03 \pm 1.33$ & $-0.31 \dagger \pm 0.74$ & $4.84 \pm 1.48$ & $-0.27 \pm 0.70$ & $5.08 \pm 1.14$ & $-0.52 \pm 0.67$ & $5.14 \pm 1.44$ & $-0.11 \pm 0.82$ & .793 & .225 \\
\hline LDL/HDL & $3.31 \pm 1.07$ & $-0.19^{*} \pm 0.65$ & $3.09 \pm 1.11$ & $-0.172 \pm 0.53$ & $3.40 \pm 0.88$ & $-0.38 \pm 0.58$ & $3.42 \pm 1.25$ & $0.01 \pm 0.80$ & .629 & .194 \\
\hline Apo B/Al & $0.90 \pm 0.28$ & $-0.04 \dagger \pm 0.12$ & $0.79 \pm 0.28$ & $-0.05 \pm 0.14$ & $0.89 \pm 0.23$ & $-0.03 \pm 0.10$ & $1.01 \pm 0.31$ & $-0.05 \pm 0.12$ & .081 & .824 \\
\hline HTGLA & $22.4 \pm 8.4$ & $-1.9 \dagger \pm 4.5$ & $26.8 \pm 7.6 \S \rrbracket$ & $-1.1 \pm 4.5$ & $20.6 \S \pm 8.9$ & $-2.4 \pm 4.00$ & $20.5 \pm 7.3 \pi$ & $-1.9 \pm 5.3$ & $.044 *$ & .716 \\
\hline
\end{tabular}

${ }^{*} P<.05, \dagger P<.01, \mp P<.001$, significance of $\Delta$ within group based on paired t test.

§Indicates $\Delta$ in E2/E3 is different from E3/E3, $P<0.06 ;$ \ $\Delta$ in E2/E3 is different from E4/E3, $P<.06 ; \| \Delta$ in E2/E3 is different from $\Delta$ in E4/E3, $P<$ .01 , based on least square differences.

plasma Apo E is associated with the HDL fraction. ${ }^{25}$ Apo E levels are lower in Apo E 4 subjects, ${ }^{22}$ possibly because the reduced adherence of Apo E 4 to the HDL particle produces more rapid transfer to lower density lipoproteins and more rapid hepatic clearance of both the lipoproteins and Apo E. ${ }^{26}$ Lower Apo E levels could reduce the VLDL-LPL interaction, thereby reducing cholesterol delivery to HDL during lipolysis. This would mitigate the increase in HDL-C with exercise training.

Collectively, these statistically nonsignificant differences in the LDL and HDL response to exercise training among the genotypes produced significantly greater reductions in the $\mathrm{TC} /$ HDL-C, LDL-C/HDL-C, and Apo B/AI rations in the Apo E $3 / 3$ homozygotes than in the Apo E 3/4 subjects implying a greater exercise training effect on coronary artery disease (CAD) risk. We believe this is the first study to document prospectively that the beneficial effects of exercise training on CAD risk may vary by genotype. Qualitatively similar, but qualitatively smaller, reductions of these parameters in the Apo E 2/3 subjects were not significantly different from the Apo E $3 / 3$ group.
Interestingly, the most significant increases in HDL-C occurred in the Apo E genotype that experienced the smallest increase in exercise capacity. This smaller increase in $\mathrm{VO}_{2} \max$ in the Apo E 3/3 subjects was totally unexpected and has not, to our knowledge, been reported previously. Nevertheless, this effect was highly significant, occurred in the entire cohort, as well as the male and female subgroups, and persisted despite the inclusion of multiple covariates in the analysis. Consequently, these differences in the $\mathrm{VO}_{2}$ response are unlikely to be due to chance, and we can identify no elements of study design that explain this observation.

There is animal evidence that Apo E can affect exercise performance. Exercise performance is reduced in Apo E-deficient mice. This effect is attributed to reduced muscular blood flow due to less nitric oxide (NO) production secondary to the hyperlipidemia characteristic of Apo E deficiency. ${ }^{27}$ In contrast to our results, however, exercise performance increased similarly with exercise training in both Apo E-deficient and normal mice, although performance in Apo E-deficient mice after training remains below that of trained normal animals. ${ }^{28}$ Apo E-deficient mice also demonstrate less collateral vessel devel-

Table 7. Baseline Physiological Characteristics, and Change With Training ( $\Delta$ ), in All Women and by Apo E Genotype (means \pm SD)

\begin{tabular}{|c|c|c|c|c|c|c|c|c|c|c|}
\hline & \multicolumn{2}{|c|}{ All Women } & \multicolumn{2}{|c|}{$2 / 3(n=23)$} & \multicolumn{2}{|c|}{$3 / 3(n=21)$} & \multicolumn{2}{|c|}{$4 / 3(n=19)$} & \multirow{2}{*}{$\begin{array}{c}\text { ANOVA on } \\
\text { Baseline, } \\
P \text { Value }\end{array}$} & \multirow{2}{*}{$\begin{array}{c}\text { ANOVA } \\
\text { or } \\
\text { ANCOVA } \\
\text { on } \\
\text { Changes, } \\
P \text { Value }\end{array}$} \\
\hline & Pre & $\Delta$ & Pre & $\Delta$ & Pre & $\Delta$ & Pre & $\Delta$ & & \\
\hline Age $(y r)$ & $39.4 \pm 11.6$ & & $40.3 \pm 10.3$ & - & $43.0 \pm 14.2$ & - & $34.8 \pm 8.6$ & - & .081 & - \\
\hline Body weight $(\mathrm{kg})$ & $71.6 \pm 14.2$ & $-0.7 \pm 2.7^{*}$ & $72.6 \pm 14.3$ & $-0.2 \pm 2.8$ & $70.4 \pm 14.4$ & $-1.2 \pm 3.1$ & $71.6 \pm 14.6$ & $-0.7 \pm 1.8$ & .889 & .428 \\
\hline$\%$ Body fat & $24.1 \pm 5.7$ & $-1.3 \pm 2.9 \dagger$ & $24.3 \pm 5.1$ & $-1.1 \pm 2.4$ & $24.1 \pm 6.4$ & $-1.5 \pm 3.0$ & $24.0 \pm 5.9$ & $-1.5 \pm 3.5$ & .990 & .873 \\
\hline WHR & $0.77 \pm 0.05$ & $-0.00 \pm 0.03$ & $0.76 \pm 0.04$ & $-0.01 \pm 0.02$ & $0.77 \pm 0.06$ & $-0.00 \pm 0.03$ & $0.78 \pm 0.05$ & $-0.00 \pm 0.03$ & .592 & .142 \\
\hline $\begin{array}{l}\mathrm{VO}_{2} \max \\
\left(\mathrm{L} \mathrm{O}_{2} / \mathrm{min}\right)\end{array}$ & $1.96 \pm 0.40$ & $0.14 \pm 0.23 \ddagger$ & $1.90 \pm 0.41$ & $0.17 \pm 0.22$ & $1.96 \pm 0.39$ & $0.03 \S \pm 0.16$ & $2.02 \pm 0.39$ & $0.23 \pm 0.25 \S$ & .602 & $.014^{*}$ \\
\hline $\begin{array}{l}\mathrm{VO}_{2} \max \\
\left(\mathrm{mL} \mathrm{O}_{2} / \mathrm{kg} / \mathrm{min}\right)\end{array}$ & $27.9 \pm 6.2$ & $2.2 \pm 3.3 \ddagger$ & $26.42 \pm 5.9$ & $2.2 \pm 3.2$ & $28.9 \pm 7.0$ & $0.8 \S \pm 2.5$ & $28.8 \pm 7.8$ & $3.7 \pm 3.7 \S$ & .357 & $.025^{*}$ \\
\hline
\end{tabular}

Significance of change within group, based on paired $t$ test: ${ }^{*} P<.05, \uparrow P<.001, \ddagger P<.0001$.

Significance of change between groups, based on Bonferroni corrected pairwise differences, Tukey's HSD, or Scheffé post hoc tests: §change in E3/E3 significantly different from change in E4/E3, $P<0.05$. 
Table 8. Baseline Lipid Concentrations, PH LPLA, and Change With Training $(\Delta)$ in Women (means \pm SD)

\begin{tabular}{|c|c|c|c|c|c|c|c|c|c|c|}
\hline & \multicolumn{2}{|c|}{ All Women } & \multicolumn{2}{|c|}{$2 / 3(n=22)$} & \multicolumn{2}{|c|}{$3 / 3(n=19)$} & \multicolumn{2}{|c|}{$4 / 3(n=19)$} & \multirow{2}{*}{$\begin{array}{l}\text { ANOVA on } \\
\text { Pre, } P \\
\text { Value }\end{array}$} & \multirow{2}{*}{$\begin{array}{c}\text { ANOVA } \\
\text { or } \\
\text { ANCOVA } \\
\text { on } \\
\text { Changes, } \\
P \text { Value }\end{array}$} \\
\hline & Pre & $\Delta$ & Pre & $\Delta$ & Pre & $\Delta$ & Pre & $\Delta$ & & \\
\hline TC & $202.9 \pm 36.3$ & $-0.4 \pm 22.5$ & $193.7 \pm 36.9$ & $-1.2 \pm 20.0$ & $211.8 \pm 39.2$ & $-1.7 \pm 23.2$ & $204.7 \pm 31.8$ & $1.8 \pm 25.3$ & .276 & .871 \\
\hline TG & $117.0 \pm 60.0$ & $-10.7 \pm 42.8^{*}$ & $123.0 \pm 69.3$ & $-7.9 \pm 57.2$ & $120.1 \pm 58.3$ & $-10.5 \pm 34.7$ & $106.9 \pm 51.2$ & $-14.1 \pm 30.9$ & .672 & .902 \\
\hline LDL-C & $127.1 \pm 31.9$ & $1.7 \pm 20.1$ & $116.7 \pm 29.7$ & $1.3 \pm 19.7$ & $134.6 \pm 32.9$ & $-1.6 \pm 17.7$ & $131.7 \pm 31.9$ & $5.4 \pm 23.0$ & .152 & .569 \\
\hline Apo B & $115.6 \pm 27.7$ & $0.1 \pm 14.0$ & $107.7 \pm 26.0$ & $-1.3 \pm 14.7$ & $122.0 \pm 30.6$ & $0.3 \pm 11.3$ & $118.3 \pm 25.9$ & $1.4 \pm 16.0$ & .228 & .829 \\
\hline HDL-C & $52.4 \pm 10.8$ & $0.0 \pm 6.0$ & $52.4 \pm 10.9$ & $-1.1 \pm 5.3$ & $53.2 \pm 11.1$ & $2.0 \pm 6.4$ & $51.6 \pm 11.1$ & $-0.7 \pm 6.1$ & .901 & .229 \\
\hline $\mathrm{HDL}_{2}-\mathrm{C}$ & $14.8 \pm 8.5$ & $-0.3 \pm 4.9$ & $15.3 \pm 8.3$ & $-1.3 \pm 4.7$ & $14.4 \pm 9.6$ & $1.5 \pm 5.1$ & $14.6 \pm 7.8$ & $-1.0 \pm 4.5$ & .946 & .160 \\
\hline $\mathrm{HDL}_{3}-\mathrm{C}$ & $34.4 \pm 5.9$ & $0.67 \pm 5.4$ & $33.8 \pm 4.8$ & $0.91 \pm 4.9$ & $35.3 \pm 6.3$ & $0.47 \pm 7.3$ & $34.2 \pm 6.8$ & $0.62 \pm 3.8$ & .714 & .969 \\
\hline Apo Al & $165.9 \pm 32.1$ & $0.3 \pm 24.4$ & $167.9 \pm 34.7$ & $2.6 \pm 20.1$ & $169.7 \pm 37.4$ & $2.8 \pm 15.7$ & $159.8 \pm 22.4$ & $-5.1 \pm 34.4$ & .603 & .525 \\
\hline $\mathrm{TC} / \mathrm{HDL}$ & $4.05 \pm 1.21$ & $-0.03 \pm 0.49$ & $3.83 \pm 1.03$ & $0.03 \pm 0.59$ & $4.15 \pm 1.31$ & $-0.17 \pm 0.46$ & $4.19 \pm 1.32$ & $-0.05 \pm 0.38$ & .571 & .320 \\
\hline LDL/HDL & $2.57 \pm 1.00$ & $0.01 \pm 0.41$ & $2.33 \pm 0.83$ & $0.04 \pm 0.46$ & $2.66 \pm 1.09$ & $-0.10 \pm 0.37$ & $2.73 \pm 1.08$ & $0.11 \pm 0.38$ & .328 & .280 \\
\hline $\begin{array}{c}\text { Ratio Apo } \\
\text { B/AI }\end{array}$ & $0.73 \pm 0.26$ & $0.00 \pm 0.18$ & $0.68 \pm 0.28$ & $-0.04 \pm 0.22$ & $0.75 \pm 0.28$ & $-0.01 \pm 0.07$ & $0.76 \pm 0.22$ & $-0.05 \pm 0.20$ & .578 & .320 \\
\hline HTGLA & $14.5 \pm 6.2$ & $-0.3 \pm 4.2$ & $13.9 \pm 5.2$ & $0.3 \pm 5.4$ & $12.8 \pm 5.6$ & $-0.3 \pm 2.8$ & $16.7 \pm 7.2$ & $-0.9 \pm 4.1$ & .130 & .589 \\
\hline
\end{tabular}

${ }^{*} P<.05$ significance of $\Delta$ within group based on paired $t$ test.

$\dagger \Delta$ in E3/E3 is different from $\Delta$ in E2/E3, $P<0.05 ; \ddagger \Delta$ in E3/E3 is different from $\Delta$ in E4/E3, $P<.05$ based on Bonferroni corrected post hoc tests.

opment in response to ischemia. ${ }^{29}$ This is associated with reduced vascular endothelial growth factor (VEGF) synthesis, again attributed to hyperlipidemia in the Apo E-deficient animals. Skeletal muscle capillary density also decreases with increasing LDL-C and Apo B concentrations in humans, ${ }^{30}$ suggesting that serum lipids may also affect skeletal muscle perfusion, and possibly performance, in man. Such observations are unlikely to explain the present results, however, because the smallest increases in exercise capacity occurred in the Apo E 3/3 subjects, the group with the putatively most beneficial changes in serum lipids.

Consequently, the effect of Apo E genotype on exercise capacity may have been mediated by more direct effects on other tissues such as skeletal muscle. Apo E mRNA is expressed in skeletal muscle and appears to be most abundant at neuromuscular junctions. ${ }^{31}$ Its role in muscle function is not clear, but Apo E-deficient animals develop abnormal mitochondria as well as sacroplasmic inclusions that react with an antibody to a fragment of the Alzheimer's cerebrovascular amyloid peptide. ${ }^{32}$ Alzheimer's disease is also related to Apo E by virtue of its increased prevalence in Apo E4 homozygotes. ${ }^{33}$ Apo $\mathrm{E}$ in the nervous system appears to function in neuronal repair after injury. ${ }^{34}$ Since exercise training is a process of repetitive muscle injury, repair, and hypertrophy, it is conceivable that the Apo E effect on exercise performance is mediated by a role, as yet undefined, in the muscle injury and repair process. If true, this will expand the relationship between lipoprotein metabolism and muscular function, although such a possibility is highly speculative.

Several other genetic factors have been linked to exercise performance. A 287-bp deletion (D) in the gene for angiotensin-converting enzyme (ACE) is associated with higher serum ACE levels in DD subjects than in those with the insertion (I) genotype. ${ }^{35}$ Subjects with the I allele demonstrate enhanced endurance performance in some, ${ }^{36-38}$ but not all, ${ }^{39}$ cross-sectional studies and a greater increase in forearm flexion ability with a fixed weight with exercise training. ${ }^{38}$ A genetic polymorphism in muscle-specific creatinine kinase gene has also been associated with greater increases in endurance capacity with exercise training. ${ }^{40}$

There are limitations to the present study that could have affected the $\mathrm{VO}_{2}$ results. The study was not originally designed to examine the influence of Apo $\mathrm{E}$ on exercise performance. Nevertheless, all of the exercise testing was performed using standard techniques in academic exercise physiology departments. Testing equipment was calibrated using gases of known composition on each testing day. Possible errors in $\mathrm{VO}_{2}$ measurements, although unlikely, should have equally affected all Apo E genotypes. The physiologic changes with exercise training in the present study were small. $\mathrm{VO}_{2}$ max increased by only $10 \%$ in the men and $8 \%$ in the women. This is less than the $15 \%$ and $19 \%$ increases in $\mathrm{VO}_{2}$ max observed in men and women, respectively, in the Health, Risk Factors, Exercise Training and Genetics (HERITAGE) study, a 20-week, multicenter study of 745 subjects designed to examine genetic determinants of the training response. ${ }^{41}$ On the other hand, TGs decreased $18 \%$ and $8 \%$ for our men and women, respectively, and HDL-C changed by $3 \%$ and $-1 \%$. In the HERITAGE study, TGs decreased only $5 \%$ in the men and $2 \%$ in the women. The increase in HDL-C for HERITAGE subjects averaged only $3 \%$ or 1.4 and 1.1 $\mathrm{mg} / \mathrm{dL}$ for men and women. Consequently, the overall physiologic changes achieved with the present study are comparable to those in similar programs. The present study also lacked a control group. We used an uncontrolled design because the primary study goal was to compare the lipid response to training among the Apo E genotypes and recruiting a control group for each genotype would have required genetically screening more than 500 additional subjects. Some of the serum lipid "exercise training effect" in the present study could represent an acute effect produced by recent exercise and not a consequence of more persistent metabolic changes induced by exercise training. The acute exercise effect primarily affects serum TGs, ${ }^{41}$ however, so the different responses in the cholesterol/ HDL ratios among the Apo E genotypes are likely to persist even when lipids are not measured within 24 hours of exercise. Finally, we cannot exclude the possibility that some other 
genetic factor associated with Apo E variants is responsible for the differences in the $\mathrm{VO}_{2}$ max response.

In conclusion, the present results demonstrate that Apo E polymorphisms affect the lipid response to exercise training in healthy, normolipidemic subjects. Reductions in LDL-C and increases in HDL-C are greater in Apo E 3 homozygotes producing significantly greater reductions with exercise training in several common markers of CAD risk, including the ratios of TC and LDL-C to HDL. In addition, the present results provide evidence that Apo E genotype affects the change in exercise performance with exercise training. Additional studies are needed to confirm this finding and, if confirmed, to define the mechanism for this effect. The qualitatively small changes in serum lipids among the Apo E genotype groups have little physiological significance for individual subjects, but are direct support of the concept that genetic variability affects the physiological responses to exercise. These small differences among genotypes could have clinically greater significance for population studies.

\section{ACKNOWLEDGMENT}

We thank the students and technicians at the participating institutions for their efforts and the subjects for their enthusiastic participation.

\section{REFERENCES}

1. Thompson PD, Cullinane EM, Sady SP, et al: High density lipoprotein metabolism in endurance athletes and sedentary men. Circulation 84:140-152, 1991

2. Thompson PD: What do muscles have to do with lipoproteins? Circulation 81:1428-1430, 1990

3. Oscai LB, Essig DA, Palmer WK: Lipase regulation of muscle triglyceride hydrolysis. J Appl Physiol 69:1571-1577, 1990

4. Goldberg IJ: Lipoprotein lipase and lipolysis: Central roles in lipoprotein metabolism and atherogenesis. J Lipid Res 37:693-707, 1996

5. Tran ZV, Weltman A: Differential effects of exercise on serum lipid and lipoprotein levels seen with changes in body weight. A meta-analysis. JAMA 254:919-924, 1985

6. Thompson PD, Rader DJ: Does exercise increase HDL cholesterol in those who need it the most? Arterioscler Thromb Vasc Biol 21:1097-1098, 2001

7. Havel RJ, Yamada N, Shames DM: Role of apolipoprotein E in lipoprotein metabolism. Am Heart J 113:470-474, 1987

8. Takahashi S, Suzuki J, Kohno M, et al: Enhancement of the binding of triglyceride-rich lipoproteins to the very low density lipoprotein receptor by apolipoprotein $\mathrm{E}$ and lipoprotein lipase. J Biol Chem 270:15747-15754, 1995

9. Breslow JL: Genetic basis of lipoprotein disorders. J Clin Invest 84:373-380, 1989

10. Dallongeville J, Lussier-Cacan S, Davignon J: Modulation of plasma triglyceride levels by apoE phenotype: A meta-analysis. J Lipid Res 33:447-454, 1992

11. Thompson PD, Jeffery RW, Wing RR, et al: Unexpected decrease in plasma high density lipoprotein cholesterol with weight loss. Am J Clin Nutr 32:2016-2021, 1979

12. Richard P, Thomas G, de Zulueta MP, et al: Common and rare genotypes of human apolipoprotein $\mathrm{E}$ determined by specific restriction profiles of polymerase chain reaction-amplified DNA. Clin Chem 40: 24-29, 1994

13. Cullinane EM, Yurgalevitch SM, Saritelli AL, et al: Variations in plasma volume affect total and low-density lipoprotein cholesterol concentrations during the menstrual cycle. Metabolism 44:965-971, 1995

14. Thompson PD, Yurgalevitch SM, Flynn MM, et al: Effect of prolonged exercise training without weight loss on high-density lipoprotein metabolism in overweight men. Metabolism 46:217-223, 1997

15. Jackson AS, Pollock ML: Generalized equations for predicting body density of men. Br J Nutr 40:497-504, 1978

16. Jackson AS, Pollock ML, Ward A: Generalized equations for predicting body density of women. Med Sci Sports Exerc 12:175-181, 1980

17. Pollock ML, Fox SM: Exercise in Health and Disease: Evalua- tion and Prescription for Prevention and Rehabilitation. Philadelphia, PA, Saunders, 1984

18. Nutrient Data System. Minneapolis, MN, University of Minnesota, 2002

19. Larkin FA, Metzner HL, Guire KE: Comparison of three consecutive-day and three random-day records of dietary intake. J Am Diet Assoc 91:1538-1542, 1991

20. Thompson PD, Moyna NM, Tsongalis G: The effect of apoE genotype on the lipid response to exercise training. Med Sci Sports Exerc 31:S135, 1999 (suppl, abstr)

21. Lehtimaki T, Frankberg-Lakkala H, Solakivi T, et al: The effect of short-term fasting, apolipoprotein E gene polymorphism, and sex on plasma lipids. Am J Clin Nutr 66:599-605, 1997

22. Boerwinkle E, Hixson JE: Genes and normal lipid variation. Curr Opin Lipidol 1:151-159, 1990

23. Nemeth A, Szakmary K, Kramer J, et al: Apolipoprotein E and complement $\mathrm{C} 3$ polymorphism and their role in the response to gemfibrozil and low fat low cholesterol therapy. Eur J Clin Chem Clin Biochem 33:799-804, 1995

24. Wilson PW, Schaefer EJ, Larson MG, et al: Apolipoprotein E alleles and risk of coronary disease. A meta-analysis. Arterioscler Thromb Vasc Biol 16:1250-1255, 1996

25. Avogaro P, Bittolo BG, Cazzolato G, et al: Distribution and concentration of apolipoprotein $\mathrm{E}$ in high and low density lipoproteins. Ric Clin Lab 13:431-436, 1983

26. Weintraub MS, Eisenberg S, Breslow JL: Dietary fat clearance in normal subjects is regulated by genetic variation in apolipoprotein $\mathrm{E}$. J Clin Invest 80:1571-1577, 1987

27. Maxwell AJ, Schauble E, Bernstein D, et al: Limb blood flow during exercise is dependent on nitric oxide. Circulation 98:369-374, 1998

28. Niebauer J, Maxwell AJ, Lin PS, et al: Impaired aerobic capacity in hypercholesterolemic mice: Partial reversal by exercise training. Am J Physiol 276:H1346-H1354, 1999

29. Couffinhal T, Silver M, Kearney M, et al: Impaired collateral vessel development associated with reduced expression of vascular endothelial growth factor in ApoE-/- mice. Circulation 99:3188-3198, 1999

30. Shono N, Mizuno M, Nishida $\mathrm{H}$, et al: Decreased skeletal muscle capillary density is related to higher serum levels of low-density lipoprotein cholesterol and apolipoprotein B in men. Metabolism 48: 1267-1271, 1999

31. Akaaboune M, Villanova M, Festoff BW, et al: Apolipoprotein $\mathrm{E}$ expression at neuromuscular junctions in mouse, rat and human skeletal muscle. FEBS Lett 351:246-248, 1994

32. Robertson TA, Dutton NS, Martins RN, et al: Comparison of astrocytic and myocytic metabolic dysregulation in apolipoprotein $\mathrm{E}$ deficient and human apolipoprotein E transgenic mice. Neuroscience 98:353-359, 2000 
33. Saunders AM, Strittmatter WJ, Schmechel D, et al: Association of apolipoprotein $\mathrm{E}$ allele epsilon 4 with late-onset familial and sporadic Alzheimer's disease. Neurology 43:1467-1472, 1993

34. Vancea JE, Campenotb RB, Vancec DE: The synthesis and transport of lipids for axonal growth and nerve regeneration. Biochim Biophys Acta 1486:84-96, 2000

35. Samani NJ, Thompson JR, O'Toole L, et al: A meta-analysis of the association of the deletion allele of the angiotensin-converting enzyme gene with myocardial infarction. Circulation 94:708-712, 1996

36. Hagberg JM, Ferrell RE, McCole SD, et al: $\mathrm{VO}_{2} \max$ is associated with ACE genotype in postmenopausal women. J Appl Physiol 85:1842-1846, 1998

37. Gayagay G, Yu B, Hambly B, et al: Elite endurance athletes and the ACE I allele-The role of genes in athletic performance. Hum Genet 103:48-50, 1998

38. Montgomery HE, Marshall R, Hemingway H, et al: Human gene for physical performance. Nature 393:221-222, 1998

39. Rankinen T, Wolfarth B, Simoneau JA, et al: No association between the angiotensin-converting enzyme ID polymorphism and elite endurance athlete status. J Appl Physiol 88:1571-1575, 2000

40. Rivera MA, Perusse L, Simoneau JA, et al: Linkage between a muscle-specific $\mathrm{CK}$ gene marker and $\mathrm{VO}_{2} \max$ in the HERITAGE Family Study. Med Sci Sports Exerc 31:698-701, 1999

41. Leon AS, Rice T, Mandel S, et al: Blood lipid response to 20 weeks of supervised exercise in a large biracial population: The HERITAGE Family Study. Metabolism 49:513-520, 2000 